\title{
Phonon plasmon interaction in ternary group-III-nitrides
}

Cite as: Appl. Phys. Lett. 101, 041909 (2012); https://doi.org/10.1063/1.4739415

Submitted: 10 February 2012 . Accepted: 12 July 2012 . Published Online: 26 July 2012

Ronny Kirste, Stefan Mohn, Markus R. Wagner, Juan S. Reparaz, and Axel Hoffmann

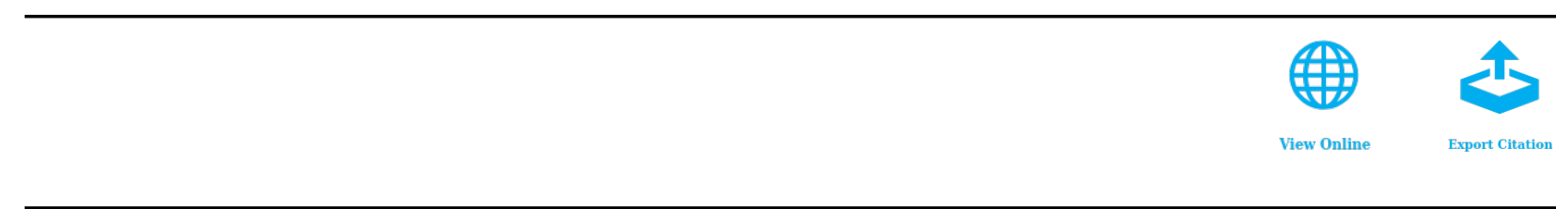

\section{ARTICLES YOU MAY BE INTERESTED IN}

Unique optical properties of $\mathrm{AlGaN}$ alloys and related ultraviolet emitters

Applied Physics Letters 84, 5264 (2004); https://doi.org/10.1063/1.1765208

Polarization-enhanced Mg doping of AlGaN/GaN superlattices

Applied Physics Letters 75, 2444 (1999); https://doi.org/10.1063/1.125042

Effect of doping and polarization on carrier collection in InGaN quantum well solar cells Applied Physics Letters 98, 243507 (2011); https://doi.org/10.1063/1.3595487

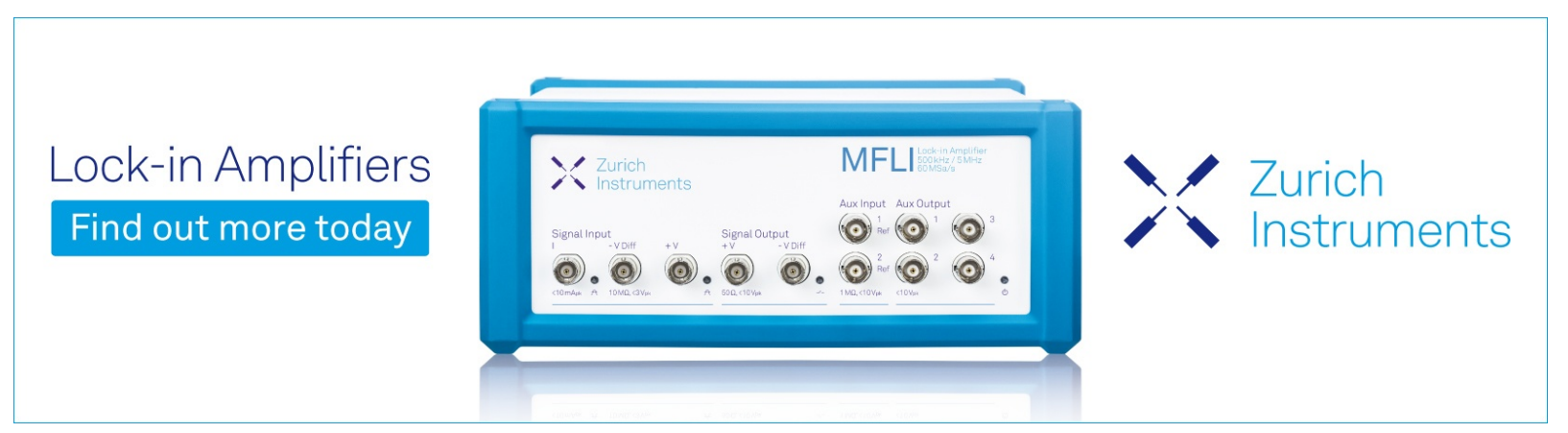




\title{
Phonon plasmon interaction in ternary group-III-nitrides
}

\author{
Ronny Kirste, Stefan Mohn, Markus R. Wagner, Juan S. Reparaz, and Axel Hoffmann \\ TU Berlin, Institut für Festkörperphysik, Hardenbergstraße 36, 10623 Berlin, Germany
}

(Received 10 February 2012; accepted 12 July 2012; published online 26 July 2012)

\begin{abstract}
Phonon-plasmon-coupling in the ternary group-III-nitrides InGaN and AlGaN is investigated experimentally and theoretically. Based on the observation of broadening and shifting of the $A_{1}(L O)$ mode in AlGaN upon Si-doping, a lineshape analysis was performed to determine the carrier concentration. The results obtained by this method are in excellent agreement to those from Hall measurements, confirming the validity of the employed model. Finally, neglecting phonon and plasmon damping, the Raman shift of the $\mathrm{A}_{1}(\mathrm{LO})$ mode in dependence of the carrier concentration for $\mathrm{AlGaN}$ and $\mathrm{InGaN}$ is calculated. This enables a fast and contactless determination of carrier concentrations in the future. (C) 2012 American Institute of Physics. [http://dx.doi.org/10.1063/1.4739415]
\end{abstract}

Over the last few years, $\mathrm{AlGaN}$ and $\mathrm{InGaN}$ have fortified their position as the most important materials for visible to ultraviolet-optoelectronic devices. ${ }^{1,2}$ Although significant progress has been made in the field of light emitting diodes (LEDs) and laser diodes, the efficiency of such devices, especially in the mid- and high-range Al-content region $\left(\lambda_{\text {gap }}<310 \mathrm{~nm}\right)$, is still well below the theoretically expected values and far from what is needed for broad commercial application. ${ }^{3,4}$ The main obstacles that have to be overcome to achieve high quality and highly efficient LEDs and laser diodes are strain fields due to lattice mismatch between InGaN/AlGaN layers and substrate, the efficiency droop, p-doping of $\mathrm{AlGaN}$, and a profound understanding and reduction of the non-radiative recombination processes.

Raman spectroscopy is a powerful method to investigate the structural and optical properties of semiconductor epilayers. ${ }^{5-8}$ Furthermore, it can be used to study the electronic properties of crystals by exploiting the coupling of longitudinal phonons to the electron plasma (LOPC). ${ }^{9-11}$ The advantages of Raman spectroscopy over Hall measurements for the determination of carrier concentration and mobility are obvious: it is non-destructive, fast, enables measurements with high spatial resolution, and can even be used in-situ. ${ }^{12}$ Especially in GaN, very impressive results have been demonstrated, whereby the charge carrier concentration and mobility have been determined with an accuracy comparable to that of Hall measurements. ${ }^{13}$ Although LOPC was also observed in AlN and InN, it is found to be much weaker in these systems. ${ }^{14,15}$ For ternary group III-nitrides, only little work has been done, so far. Very recently, results for AlGaN were presented, demonstrating the validity and usefulness of the LOPC-theory for AlGaN (Al-content around 68\%, carrier concentration $\left.10^{17} \mathrm{~cm}^{-3}\right) .{ }^{16}$ However, no data are available for other Al-contents or InGaN at all.

In this contribution, the effect of silicon doping on AlGaN with $10 \%$ and $28 \%$ nominal Al-content is investigated experimentally and theoretically. Additionally, LOPC in InGaN is investigated theoretically. Samples are characterized with Hall measurements and Raman spectroscopy. It is demonstrated that $\mathrm{Si}$ doping leads to a relaxation of compressive strain and a shift and broadening of the longitudinal optical (LO) Raman mode. This behavior of the LO-mode is explained by LOPC. Using the model of Irmer et al., ${ }^{10}$ the line shape of the LO-mode is fitted and charge carrier concentrations of the epilayers are determined. Results are compared to those of Hall measurements and a good agreement is found. Finally, using a model that neglects phonon and plasmon damping, the Raman shift of the LO-mode depending on the carrier concentration is calculated. This calculation is performed for $\mathrm{AlGaN}$ and $\mathrm{InGaN}$. An excellent agreement between the results using this model and the experimentally determined carrier concentrations in this work and a recently published work by Kim et al. is found demonstrating the strength of this method. ${ }^{16}$ The presented work will help to establish Raman spectroscopy as a standard tool for the determination of carrier concentrations in AlGaN and InGaN, independently or complementary to Hall measurements.

Not intentionally doped (nid) $1000 \mathrm{~nm}$ thick AlGaN layers with $10 \%$ and $28 \% \mathrm{Al}$-content were grown by metal organic chemical vapor deposition (MOCVD) on AlN/ sapphire substrates. For each undoped sample, a complementary sample doped with silicon was prepared. Room temperature Hall measurements were used to determine the charge carrier concentration and mobility. Raman spectra were recorded using a Dilor XY system with the $488 \mathrm{~nm}$ line of an Ar-ion laser for excitation, a $2 \mathrm{~m}$ double monochromator for dispersion and a liquid nitrogen cooled charged-coupled device (CCD) for detection. The fitting and simulation of the spectra were performed using Mathematica.

Fig. 1 shows Raman spectra of doped and undoped $\mathrm{AlGaN}$ with $10 \%$ and $28 \% \mathrm{Al}$-content recorded in the $\mathrm{z}(\mathrm{xx}) \mathrm{z}$ geometry. In addition to the Raman modes of the substrate (AlN, sapphire), two peaks arising from the AlGaN layers can be observed for all samples: ${ }^{12}$ the $\mathrm{E}_{2}$ (high) mode at around $570-580 \mathrm{~cm}^{-1}$ and the $\mathrm{A}_{1}(\mathrm{LO})$ mode at $760-810 \mathrm{~cm}^{-1}$. The $\mathrm{E}_{2}$ (high) mode is non-polar and consequently a good indicator for strain in the samples. For the undoped samples, a compressive strain is observed, which leads to a shift of around $3 \mathrm{~cm}^{-1}$ to higher energies, compared to the expected value for relaxed AlGaN. ${ }^{12}$ Apparently, the $\mathrm{E}_{2}$ (high) shifts, independent of the Al-content, to lower energies for the Si-doped samples compared to the undoped samples. Thus, the Si-doping leads to a relaxation of compressive strain as it was observed before for AlN, GaN, and AlGaN. ${ }^{17-19}$ In contrast to the $\mathrm{E}_{2}$ (high) mode, the $\mathrm{A}_{1}(\mathrm{LO})$ mode is polar: plasmons and phonons may 


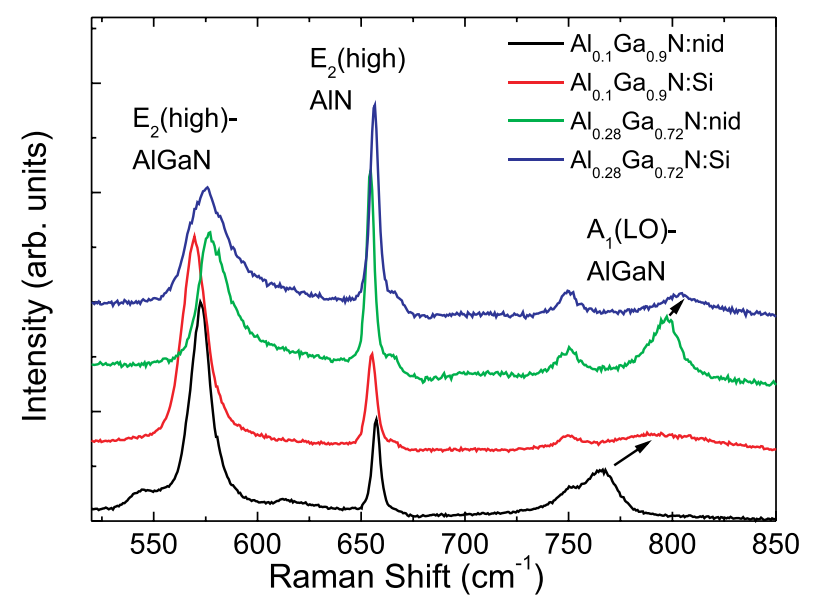

FIG. 1. First order Raman spectra of undoped and Si-doped hexagonal AlGaN with $10 \%$ and $28 \%$ Al-content, respectively.

couple and lead to a shift and broadening of the $\mathrm{A}_{1}(\mathrm{LO})$ mode. Fig. 2 displays Raman spectra in the area of the $\mathrm{A}_{1}(\mathrm{LO})$ mode for an undoped and a Si-doped sample with $10 \% \mathrm{Al}$-content. Each spectrum consists of a peak at around $750 \mathrm{~cm}^{-1}$, which is related to the sapphire substrate and the $\mathrm{A}_{1}(\mathrm{LO})$ peak. It is obvious that the $\mathrm{A}_{1}(\mathrm{LO})$ mode shifts to higher energies and broadens upon Si-doping. Such a behavior in doped samples was observed in GaN and AIN before ${ }^{9,14}$ and is explained by longitudinal optical phonon plasmon coupling. In the case of coupled phonon plasmon modes, the LOmode splits into a so called $\mathrm{LOPC}^{-}$and $\mathrm{LOPC}^{+}$mode. At low carrier concentrations the $\mathrm{LOPC}^{-}$mode is plasmon-like while the $\mathrm{LOPC}^{+}$mode has the energy of the LO-phonons. With increasing carrier concentrations their energies increase: in the case of the $\mathrm{LOPC}^{-}$the energy increases towards that of the transversal optical (TO) phonons and in the case of the $\mathrm{LOPC}^{+}$to that of the plasmons. Following, the $\mathrm{A}_{1}(\mathrm{LO})$ will hereafter be referred as $\mathrm{LOPC}^{+}$mode.

The charge carrier concentration and mobility can be calculated from the parameters of a fit of the lineshape of the $\mathrm{LOPC}^{+}$mode. In this work, a model for binary materials is

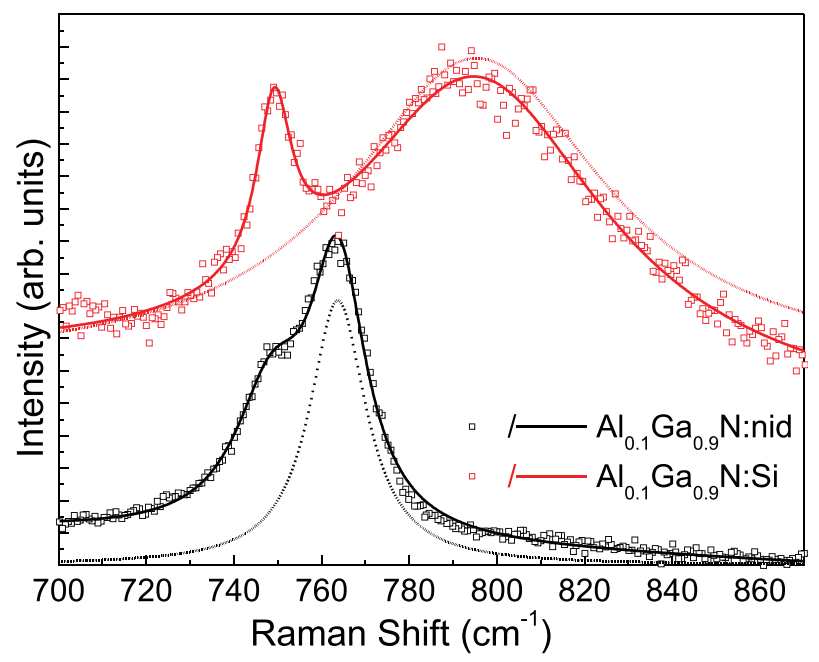

FIG. 2. Raman spectra in the area of the LO-Raman mode of undoped and Si-doped $\mathrm{Al}_{0.1} \mathrm{Ga}_{0.9} \mathrm{~N}$ (squares) and related fits (straight lines). The spectra consist of a substrate related peak around $750 \mathrm{~cm}^{-1}$, a linear background, and the $\mathrm{LOPC}^{+}$mode of $\mathrm{AlGaN}$ (dotted line). used. This is mainly motivated by the simplicity of the model, the fact that the LO-mode in AlGaN reveals a single mode behavior and the good results from its usage as presented below and before. ${ }^{16,20}$ The lineshape of the coupled modes can be described by the following expression: ${ }^{10,21}$

$$
I_{A}=\text { const } \cdot A(\omega) \cdot \operatorname{Im}\left\{-\varepsilon(\omega)^{-1}\right\} .
$$

Thereby, $\mathrm{A}(\omega)$ is related to the deformation potential and electro-optic scattering, and $\varepsilon(\omega)$ is the dielectric constant. $\mathrm{A}(\omega)$ depends on the Faust-Henry coefficient, ${ }^{21}$ the LO and TO phonon frequencies of undisturbed $\mathrm{AlGaN},{ }^{20}$ the plasma frequency $\omega_{\mathrm{p}}$, the phonon damping $\Gamma$, and the plasmon damping $\gamma$, whereby the last three variables are the fitting parameters of Eq. (1). The influence of each parameter on the Raman spectra was nicely illustrated by Park et al. for GaN. ${ }^{21}$ In addition to the measured Raman spectra, a fit of the $\mathrm{LOPC}^{+}$mode using Eq. (1) is displayed in Fig. 2 (straight line). From the plasmon frequency the carrier concentration can be calculated by using

$$
\omega_{\mathrm{p}}=\sqrt{\frac{4 \pi \mathrm{ne}^{2}}{\epsilon^{\infty} \mathrm{m}^{*}}},
$$

whereby $\mathrm{n}$ is the free carrier concentration and the other symbols have their usual meaning. Table I lists the carrier concentrations as determined by Hall measurements and the plasma frequencies and carrier concentrations as determined by the fit of the Raman spectra. A good agreement between the results from Hall- and Raman measurements is found for all samples. For the undoped samples, the carrier concentrations calculated from the Raman spectra are below or around low $10^{17} \mathrm{~cm}^{-3}$. The main reason for the deviation of the values determined by Hall measurements are the dependence of Eq. (1) on the position of the undisturbed $A_{1}(\mathrm{LO})$ and $\mathrm{A}_{1}$ (TO) modes, which are controversial in literature. ${ }^{20,22}$ The calculated values for the carrier concentrations in the doped samples differ only by a factor of $1.3-1.8$ to the measured values. These are acceptable deviations and demonstrate the high potential of this method for everyday application for the determination of carrier concentrations in AlGaN by Raman spectroscopy. In principle, it is also possible to determine the charge mobility with this method. However, similar to observations in $\mathrm{GaN}$ and $\mathrm{GaP},{ }^{10,13}$ the values calculated for the mobility for our samples differ very much from the measured values. As the main parameter for the calculation of the mobility is the plasmon damping, this deviation can be caused by any other effect that broadens the $\mathrm{LOPC}^{+}$mode, such as broadening by elastic scattering or compositional fluctuations. $^{20}$

The dielectric constant $\varepsilon(\omega)$ in Eq. (1) depends on the phonon and plasmon frequencies and damping. ${ }^{9}$ If the latter is neglected and $\varepsilon(\omega)=0$, the equation simplifies and the frequency of the $\mathrm{LOPC}^{+}$- and an $\mathrm{LOPC}^{-}$mode can be directly calculated by

$$
\omega_{L O P C \pm}^{2}=\frac{\omega_{L O}^{2}+\omega_{p}^{2}}{2} \pm \sqrt{\left[\left(\frac{\omega_{L O}^{2}+\omega_{p}^{2}}{2}\right)^{2}-\omega_{T O}^{2} \omega_{p}^{2}\right]} .
$$


TABLE I. Charge carrier concentrations as determined by Hall measurements ( $\left.\mathrm{n}_{\text {Hall }}\right)$, fitted Raman spectra using Eq. (1) ( $\left.\mathrm{n}_{\text {Raman }}\right)$, and using the undamped model as described by Eq. (4) $\left(\mathrm{n}_{\text {undamped }}\right)$. For convenience, the plasma frequency $\left(\omega_{\mathrm{p}}\right)$ and the position of the LOPC $\mathrm{C}^{+}$mode are also listed.

\begin{tabular}{lccccc}
\hline \hline Sample & $\mathrm{n}_{\text {Hall }}\left(10^{17} \mathrm{~cm}^{-3}\right)$ & $\mathrm{n}_{\text {Raman }}\left(10^{17} \mathrm{~cm}^{-3}\right)$ & $\omega_{\mathrm{p}}\left(\mathrm{cm}^{-1}\right)$ & $\mathrm{LOPC}^{+}\left(\mathrm{cm}^{-1}\right)$ & $\mathrm{n}_{\text {undamped }}\left(10^{17} \mathrm{~cm}^{-3}\right)$ \\
\hline $\mathrm{Al}_{0.1} \mathrm{Ga}_{0.9} \mathrm{~N}:$ :nid & $<1$ & 2.7 & 152 & 763 & 3 \\
$\mathrm{Al}_{0.1} \mathrm{Ga}_{0.9} \mathrm{~N}: \mathrm{Si}$ & 18 & 14 & 345 & 795 & 13 \\
$\mathrm{Al}_{0.28} \mathrm{Ga}_{0.72} \mathrm{~N}:$ :nid & $<1$ & 0.9 & 84 & 794 & $<1$ \\
$\mathrm{Al}_{0.28} \mathrm{Ga}_{0.72} \mathrm{~N}: \mathrm{Si}$ & 23 & 13 & 337 & 809 & 8 \\
\hline \hline
\end{tabular}

Here, $\omega_{\mathrm{LO}}$ and $\omega_{\mathrm{TO}}$ are the composition dependent phonon frequencies of undisturbed AlGaN or InGaN. In order to enable a fast and direct determination of the carrier concentration directly from the measured Raman spectra, without any fitting procedure, the frequencies of the $\mathrm{LOPC}^{+}$and $\mathrm{LOPC}^{-}$ mode, depending on the carrier concentration, were calculated for the whole compositional range of $\mathrm{AlGaN}$ and InGaN, as it was done before for GaN. ${ }^{9,13}$ The results of these calculations are displayed in Fig. 3. For low carrier concentrations in AlGaN as shown in Figs. 3(a) and 3(b), the position of the $\mathrm{LOPC}^{+}$modes directly scales with the Alcontent. This corresponds to the compositional dependence of the energy of the $\mathrm{A}_{1}(\mathrm{LO})$ mode. As carrier concentrations increases the $\mathrm{LOPC}^{+}$mode shifts to higher energies and becomes more plasmon-like. Thereby, the slope of the Garich branches is much steeper, so that at carrier concentration above $10^{19} \mathrm{~cm}^{-3}$ the $\mathrm{LOPC}^{+}$mode of $\mathrm{AlGaN}$ with less $\mathrm{Al}$ has a higher energy. The driving force for this behavior is the increased plasma frequency in $\mathrm{AlGaN}$ with higher Gacontent. The energy scaling of the $\mathrm{LOPC}^{-}$mode is converse
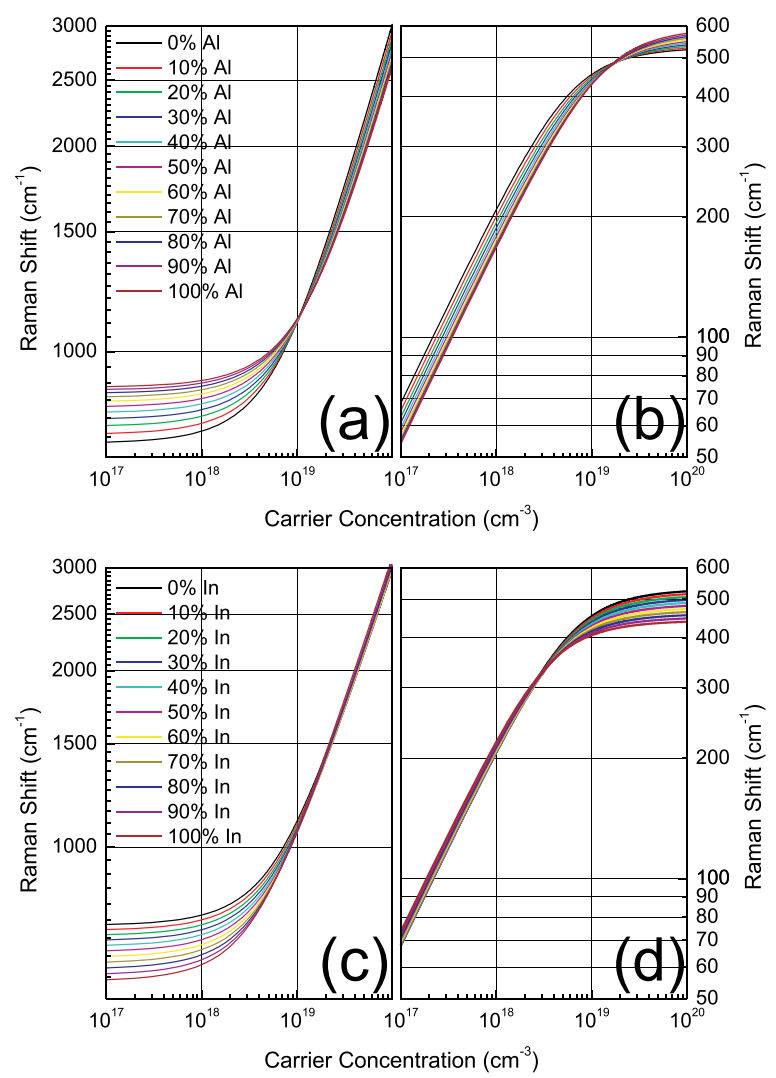

FIG. 3. Calculated Raman shift of the $\mathrm{LOPC}^{+}[(\mathrm{a}),(\mathrm{c})]$ and $\mathrm{LOPC}^{-}[(\mathrm{b})$, (d)] mode depending on the carrier concentration for $\mathrm{AlGaN}$ (top) and InGaN (bottom) with different Ga-contents. to that of the $\mathrm{LOPC}^{+}$mode, as it is plasmon-like for low carrier concentrations and phonon like for increased carrier concentrations. It is important to mention that the $\mathrm{LOPC}^{-}$mode could not be observed for our samples, probably due to the low intensity of this peak. Additionally, for ternary materials a $\mathrm{L}^{0}$ gap mode is expected. ${ }^{23}$ Typically, this mode is trapped between the energy of the LO and TO phonons of the two sublattices. However, due to the single mode behavior of the LO-modes in AlGaN, this mode is not expected for our samples.

For the $\mathrm{LOPC}^{+}$mode, which is the most commonly used mode for the determination of the carrier concentration, Eqs. (2) and (3) can be resolved to

$$
n\left(\omega_{L O P C^{+}}\right)=\frac{m^{*} \varepsilon^{\infty}}{4 \pi e^{2}}\left\{\frac{\omega_{L O P C^{+}}^{4}-\omega_{L O}^{2} \omega_{L O P C^{+}}^{2}}{\omega_{L O P C^{+}}^{2}-\omega_{T O}^{2}}\right\} .
$$

This equation is extremely useful for a fast calculation of the carrier concentration from Raman spectra. For a given Al- or In-concentration, only the known literature values of the effective mass, ${ }^{24}$ dielectric constant, ${ }^{24,25}$ and LO/TO frequency ${ }^{20,26}$ need to be inserted from literature into Eq. (4). The carrier concentration can then be directly calculated using the measured $\mathrm{LOPC}^{+}$frequency as the only input parameter.

Corrected for the influence of the strain, the measured position of the $\mathrm{LOPC}^{+}$mode was taken from Fig. 1 and used to estimate the carrier concentration using Eq. (4). Using this method, we obtained very similar results compared to those from Hall measurements and the values from the fitting that included phonon damping (Table I) for all samples, except for the doped sample with $28 \%$ nominal Al-content. For that sample, the determined charge carrier concentration was a factor of three lower than the value from Hall measurements. Additionally, Eq. (4) was used to determine the carrier concentration from samples published very recently by Kim et al. ${ }^{16}$ An excellent agreement between published values obtained by Hall measurements and those calculated using Eq. (4) is found. For example, for sample \#D in the work of Kim et al. a carrier concentration of $6 \cdot 10^{17} \mathrm{~cm}^{-3}$ is calculated which differs only very slightly from the value from Hall measurements. ${ }^{16}$ These results prove that Raman spectroscopy in connection with Eq. (4) can be broadly used to estimate carrier concentrations in $\mathrm{AlGaN}$ and $\mathrm{InGaN}$.

In summary, we presented results from Raman spectroscopy on undoped and Si-doped AlGaN layers as well as theoretical calculations for $\mathrm{AlGaN}$ and $\mathrm{InGaN}$. In addition to the relaxation of compressive strain induced by the $\mathrm{Si}$ doping of AlGaN, a strong coupling between phonons and plasmons was observed, which is expressed by a shift and 
broadening of the $A_{1}(\mathrm{LO})$ Raman mode. The lineshape of this mode was fitted using a model that was originally developed for binary materials. The fitting parameters were used to determine the carrier concentration of the layers. An excellent agreement was found between the results from Raman spectroscopy and Hall measurements. Neglecting phonon and plasmon damping, the Raman shift of the LOPC-modes depending on the carrier concentration was calculated for the whole compositional range of AlGaN and InGaN. A simple and useful equation for the direct calculation of carrier concentrations from "as measured" Raman spectra was introduced, and its ability to determine carrier concentrations over a broad range of Al-contents was demonstrated.

This work was supported by the DFG within SFB 787. The authors thank Frank Brunner et al. from the Ferdinand Braun Institut in Berlin for providing the samples and Viola Küller for providing the Hall measurement results.

${ }^{1}$ H. Hirayama, T. Yatabe, N. Noguchi, T. Ohashi, and N. Kamata, Appl. Phys. Lett. 91(7), 071901 (2007).

${ }^{2}$ S. Nakamura, T. Mukai, and M. Senoh, Appl. Phys. Lett. 64(13), 16871689 (1994).

${ }^{3}$ M. Kneissl, Z. Yang, M. Teepe, C. Knollenberg, O. Schmidt, P. Kiesel, N. M. Johnson, S. Schujman, and L. J. Schowalter, J. Appl. Phys. 101(12), 123103-123105 (2007).

${ }^{4}$ J. Zhang, H. P. Zhao, and N. Tansu, Appl. Phys. Lett. 98(17), 171111 (2011).

${ }^{5}$ A. Kaschner, U. Haboeck, M. Strassburg, M. Strassburg, G. Kaczmarczyk, A. Hoffmann, C. Thomsen, A. Zeuner, H. R. Alves, D. M. Hofmann, and B. K. Meyer, Appl. Phys. Lett. 80(11), 1909-1911 (2002).

${ }^{6}$ N. Dietz, M. Alevli, R. Atalay, G. Durkaya, R. Collazo, J. Tweedie, S. Mita, and Z. Sitar, Appl. Phys. Lett. 92(4), 041911 (2008).

${ }^{7}$ H. Shen, F. H. Pollak, and R. N. Sacks, Appl. Phys. Lett. 47(8), 891-893 (1985).
${ }^{8}$ R. Kirste, R. Collazo, G. Callsen, M. R. Wagner, T. Kure, J. S. Reparaz, S. Mita, J. Xie, A. Rice, J. Tweedie, Z. Sitar, and A. Hoffmann, J. Appl. Phys. 110(9), 093503 (2011).

${ }^{9}$ P. Perlin, J. Camassel, W. Knap, T. Taliercio, J. C. Chervin, T. Suski, I. Grzegory, and S. Porowski, Appl. Phys. Lett. 67(17), 2524-2526 (1995).

${ }^{10}$ G. Irmer, V. V. Toporov, B. H. Bairamov, and J. Monecke, Phys. Status Solidi B 119(2), 595-603 (1983).

${ }^{11}$ R. Cuscó, J. Inánez, E. Alarcón-Llardó, L. Artus, T. Yamaguchi, and Y. Nanishi, Phys. Rev. B 79(15), 155210 (2009).

${ }^{12}$ M. Kuball, Surf. Interface Anal. 31 (10), 987-999 (2001).

${ }^{13}$ H. Harima, H. Sakashita, and S. Nakashima, Mater. Sci. Forum 264-268, 1363-1366 (1998).

${ }^{14}$ M. Gómez-Gómez, A. Cros, M. Hermann, M. Stutzmann, and M. Eickhoff, Phys. Status Solidi A 206(6), 1183-1186 (2009).

${ }^{15}$ R. Cusco, N. Domenech-Amador, L. Artus, T. Gotschke, K. Jeganathan, T. Stoica, and R. Calarco, Appl. Phys. Lett. 97(22), 221906 (2010).

${ }^{16}$ J. G. Kim, A. Kimura, Y. Kamei, N. Hasuike, H. Harima, K. Kisoda, Y. Shimahara, H. Miyake, and K. Hiramatsu, Appl. Phys. Lett. 99(25), 251904 (2011).

${ }^{17}$ G. M. Prinz, M. Feneberg, M. Schirra, R. Sauer, K. Thonke, S. B. Thapa, and F. Scholz, Phys. Status Solidi (RRL) 2(5), 215-217 (2008).

${ }^{18}$ I.-H. Lee, I.-H. Choi, C. R. Lee, and S. K. Noh, Appl. Phys. Lett. 71(10), 1359-1361 (1997).

${ }^{19}$ P. Cantu, F. Wu, P. Waltereit, S. Keller, A. E. Romanov, U. K. Mishra, S. P. DenBaars, and J. S. Speck, Appl. Phys. Lett. 83(4), 674-676 (2003).

${ }^{20}$ V. Y. Davydov, I. N. Goncharuk, A. N. Smirnov, A. E. Nikolaev, W. V. Lundin, A. S. Usikov, A. A. Klochikhin, J. Aderhold, J. Graul, O. Semchinova, and H. Harima, Phys. Rev. B 65(12), 125203 (2002).

${ }^{21}$ M. Park, J. J. Cuomo, B. J. Rodriguez, W. C. Yang, R. J. Nemanich, and O. Ambacher, J. Appl. Phys. 93(12), 9542-9547 (2003).

${ }^{22}$ M. Kazan, P. Masri, and M. Sumiya, J. Appl. Phys. 100(1), 013508 (2006).

${ }^{23}$ O. K. Kim and W. G. Spitzer, Phys. Rev. B 20(8), 3258 (1979).

${ }^{24}$ B. E. Foutz, S. K. O'Leary, M. S. Shur, and L. F. Eastman, J. Appl. Phys. 85(11), 7727-7734 (1999).

${ }^{25}$ K. Takeuchi, S. Adachi, and K. Ohtsuka, J. Appl. Phys. 107(2), 023306 (2010).

${ }^{26}$ G. Kaczmarczyk, A. Kaschner, S. Reich, A. Hoffmann, C. Thomsen, D. J. As, A. P. Lima, D. Schikora, K. Lischka, R. Averbeck, and H. Riechert, Appl. Phys. Lett. 76(15), 2122-2124 (2000). 